\title{
An Empirical Analysis of Public WeChat Accounts at a University Based on the WCI Algorithm
}

\author{
Xiangpeng Yang, Yi He* \\ Central University of Finance and Economics, Beijing, China \\ Email: ^heyi@cufe.edu.cn
}

How to cite this paper: Yang, X. P., \& He, Y. (2020). An Empirical Analysis of Public WeChat Accounts at a University Based on the WCI Algorithm. Open Journal of Business and Management, 8, 2317-2325. https://doi.org/10.4236/ojbm.2020.85142

Received: July 22, 2020

Accepted: September 27, 2020

Published: September 30, 2020

Copyright $\odot 2020$ by author(s) and Scientific Research Publishing Inc. This work is licensed under the Creative Commons Attribution International License (CC BY 4.0).

http://creativecommons.org/licenses/by/4.0/

\begin{abstract}
This paper takes WeChat propagation index as the research parameter and uses the WCI specific algorithm to analyze. The analysis samples are five highly popular public WeChat accounts at the Central University of Finance and Economics. The top 50 articles with a reading index and thumbs-up rate in official WeChat accounts were selected as analysis objects. The researcher conducted a detailed analysis of the source, type, form, and release time of highly popular tweets to provide an empirical basis for the smooth development of college students' network education.
\end{abstract}

\section{Keywords}

WCI Algorithm, University WeChat Public Number, Empirical Analysis

\section{Introduction}

With the increasing popularity of WeChat every year, college students have become an important user group of the WeChat public platform. According to statistics from third-party institutions, nearly $50 \%-60 \%$ of college students pay consistent attention to WeChat public platforms. The results of a survey revealed that the WeChat public platforms that college students pay attention to mainly involve current affairs and news, gossip and commentary, travel and cuisine, and so forth (Wang, Wang, Ding, \& Wei, 2017). There are significant differences between college students' response to types of WeChat tweets, which are mainly manifested in core indicators such as the degree of recognition, the degree of acceptance, and comprehensive influence (Zhu, Zhang, \& Jin, 2016). The influ- 
ence, recognition, and acceptance of tweets directly affect the efficiency of information dissemination and indirectly affect the educational effect of new media (Matthews, 2013). The current situation of readers' feedback on WeChat public account cannot be clearly grasped, leading to the inability of the school to effectively use WeChat public account to better serve teachers and students. In view of this finding, this paper aims to understand the degree of acceptance, the degree of recognition and the influence difference. Through empirical analysis, we take universities as an example to improve the effectiveness of network education and propose some countermeasures to optimize the content of network education.

\section{Data Sources, Analysis Methods, and Indicators}

\subsection{Data Sources}

To improve the availability and validity of data, the data source of this paper consists of 3189 articles pushed by five popular public WeChat accounts at the Central University of Finance and Economics (CUFE) in 2019. These are the five most influential public accounts with the most subscriptions. These five public accounts are more representative, both official and unofficial, both graduate and undergraduate students. The five popular public WeChat platforms are "CUFE", "What CUFE", "CUFE Bengdou", "CUFE Student Affairs Office" and "CUFE Graduate Student Union."

\subsection{Data Analysis Method}

In this paper, the most authoritative index in evaluating the influence of WeChat in China, the WeChat Communication Index (WCI), serves as the research parameter. According to the setting of the WCI algorithm, the attention to WeChat content is reported in the reading index, $\mathrm{WCI}(\mathrm{R})$; the response rate is represented in the thumbs-up index, $\mathrm{WCI}(\mathrm{Z})$; and the WCI calculated by weight reflects the "heat". The attention and response rate of college students to the content of network education are important measures of the effectiveness of network education (Teresi, 2016). In the WCI algorithm, attention mainly refers to how often people read an article, which is used to measure the coverage of information transmission (Tyrer, 2019). The response rate mainly concerns the interaction between fans and public WeChat accounts after reading the article. The WCI heat calculated by combining the two can more comprehensively reflect the content and interest points to which college students pay attention (Lin \& Jian, 2019). The specific indicators and calculation formulas are shown in Table 1.

\subsection{Data Analysis Indicators}

\subsubsection{The Activity Index of Public Accounts}

This paper mainly evaluates the activity of public accounts through statistics on the total and average daily output. The total number of posts refers to the total 
Table 1. Reading index and the thumbs-up formula ${ }^{1}$.

\begin{tabular}{|c|c|c|c|}
\hline Level 1 indicators & Secondary indicators & Weight & Standardized method \\
\hline \multirow{3}{*}{$\begin{array}{l}\text { Reading index } \\
\quad(80 \%)\end{array}$} & Average daily reading $(\mathrm{R} / \mathrm{d})$ & $40 \%$ & $\ln (\mathrm{R} / \mathrm{d}+1)$ \\
\hline & Average number of readings $(\mathrm{R} / \mathrm{n})$ & $45 \%$ & $\ln (\mathrm{R} / \mathrm{n}+1)$ \\
\hline & Maximum number of readings (Rmax) & $15 \%$ & $\ln (\operatorname{Rmax}+1)$ \\
\hline \multirow{3}{*}{$\begin{array}{c}\text { Thumbs-up index } \\
(20 \%)\end{array}$} & Average thumb up number per day $(\mathrm{Z} / \mathrm{d})$ & $40 \%$ & $\ln (10 * \mathrm{Z} / \mathrm{d}+1)$ \\
\hline & Post-average thumbs-up number (Z/n) & $45 \%$ & $\ln (10 * \mathrm{Z} / \mathrm{n}+1)$ \\
\hline & Highest number of likes (Zmax) & $15 \%$ & $\ln (10 * \operatorname{Zmax}+1)$ \\
\hline \multicolumn{4}{|c|}{$\mathrm{WCI}=\{80 \% \times(40 \% \times \ln (\mathrm{R} / \mathrm{d}+1)+45 \% \times \ln (\mathrm{R} / \mathrm{d}+1)$} \\
\hline \multicolumn{4}{|c|}{$+15 \% \times \ln (\operatorname{Rmax}+1))+20 \% \times(40 \% \times \ln (10 \times \mathrm{Z} / \mathrm{d}+1)$} \\
\hline \multicolumn{4}{|c|}{$+40 \% \times \ln (10 \times \mathrm{Z} / \mathrm{n}+1)+15 \% \times \ln (10 \times \mathrm{Zmax}+1))\}^{2} \times 10$} \\
\hline
\end{tabular}

number of tweets posted on a public account during the statistical period, while the average daily posts refer to the average number of daily posts.

\subsubsection{Classification Indexes of Public Accounts' Tweets}

\section{1) Content classification index}

To study the influence characteristics of different types of WeChat official tweets, we classified the contents of 3189 tweets. Drawing lessons from Zhang Weiliang and Zhang Ping's (2017: p. 12) classification of public WeChat accounts' content, and according to the actual situation at CUFE, we divided the content of tweets into seven categories: education about mainstream values, learning and employment, campus culture, life service, news reporting, notification, voting, and investigation.

"Education about mainstream values" tweets mainly concern loving the motherland, as well as promoting social morality and quality education. The maintream values education involves ideals, beliefs, role models, and advanced figures, typical deeds, and other information; the study of employment mainly includes student training and assessment, course learning, internships and employment information; campus culture mainly entails tweets on campus history, campus life, campus events, and campus figures. Life service category mainly includes food, travel, entertainment, life service, and so forth. News category is mainly about social hot spots and campus news. Finally, notification category includes activity notifications, publicity, and so forth; poll category is tweets based on online polls and polls (Small, 2014).

\section{2) Formal classification index}

According to their manifestation, tweets were classified as photos, pictures, text, tables, audio, or video.

\section{3) Indicators of reception and acceptance}

${ }^{1}$ Qing bo index. WeChat WCI transmission index (V12.0) [EB/OL]. [2016-12-23] http://www.gsdata.cn/site/usage. 
The effective reception of a tweet means that the reader receives and reads the tweet. We used the total number of tweets read $(R)$ to evaluate this indicator. Moreover, we analyzed the thumbs-up rate $(\mathrm{Z} / \mathrm{R})$ of tweets to evaluate this index in the form of approval of, support for, and likes related to the content of tweets.

\section{Active Degree Analysis of Public Accounts}

This paper presents an analysis of the total statistics of the five hot public accounts at CUFE in 2019. We found that the group of public accounts published a large number of tweets and maintained a high degree of activity. Among them, "CUFE Graduate Student Union" published 236 articles in 2019, with an average daily publication rate of 0.65 . As the official WeChat account at CUFE, "CUFE" also maintained a high level of activity. The publication volume of the CUFE Student Affairs Office has not changed much compared with the publication volume of the "CUFE" and "CUFE Graduate Student Union". "What CUFE" has maintained a very high level of activity. Compared with the first three official Weibo posts, the number of posts has doubled, reaching 2.26 tweets per day. As an unofficial WeChat platform, "CUFE Bengdou" posted a total of 1529 tweets in 2019, with an average of 4.19 tweets per day, far higher than the four other official WeChat posts, as shown in Table 2.

\section{Analysis of Tweets of Public Accounts}

\subsection{Analysis of the Highly Read Tweets of the Sample Public Accounts}

We ranked all tweets according to their reading volume from highest to lowest and selected the top 50 tweets as the analysis sample. As shown in Figure 1, the highly read tweets come from the public accounts "CUFE", "What CUFE" and "CUFE Bengdou". The possible reason for this situation is that tweets themselves have a strong appeal, and a large number of readers follow these three public

The scale chart of the top 50 most-read tweets

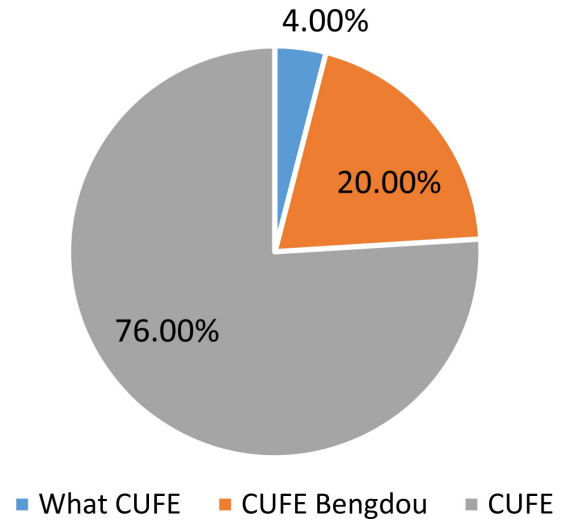

Figure 1. Proportion chart of the public accounts with the top 50 most-read tweets. 
Table 2. Activity of five popular official WeChat accounts at CUFE in 2019.

\begin{tabular}{ccc}
\hline Name of public account & Total number & $\begin{array}{c}\text { Average daily } \\
\text { publication volume }\end{array}$ \\
\hline CUFE Graduate Student Union & 236 & 0.65 \\
CUFE & 278 & 0.76 \\
CUFE Student Affairs Office & 321 & 0.88 \\
What CUFE & 825 & 2.26 \\
CUFE Bengdou & 1529 & 4.19 \\
\hline
\end{tabular}

accounts. From the perspective of reading volume alone, the top three tweets have been read 66,057; 57,771; and 56,063 times, respectively, far exceeding the total number of teachers and students. This finding indicates that the readers of these three public accounts have gone beyond the scope of the university and may include alumni, examinees, and the general public.

To be specific, the seven categories all take the form of pictures, charts, audio, or video combined with words, which also reflects the reading habits and preferences of readers. Tweets that simply use text are not attractive enough to retain readers. Among the types of tweets, campus culture is the most widely read of the top 50 tweets, accounting for $50 \%$, followed by nine studies on employment (18\%), eight news stories (16\%), six articles in the life services category (12\%), and one mainstream value category and one notification category, which account for $2 \%$ combined. As displayed in Figure 2, according to the data, students are most interested in tweets that reflect the history of the school, campus cultural life, campus events for students, and campus figures related to them. Paying attention to study and employment ranked second in terms of WeChat reading, which is consistent with the idea that students focus on these issues. The fact that the news report category ranks third reflects, to some extent, that WeChat has become an important channel for students to receive updated school information (Zhao \& Wei, 2017).

\subsection{Analysis of Tweets with a High Response Rate among Sample Public Accounts}

The ratio of the number of tweets thumbs-ups ( $\mathrm{Z}$ ) to the number of tweets read $(\mathrm{R})$ is defined as the thumbs-up rate of tweets to evaluate readers' recognition, liking, and support for a tweet. The higher the thumbs-up rate, the higher the reader's recognition of their views and values becomes. Using the WCI algorithm, we calculated the thumbs-up rate of all tweets in 2019. The top 50 tweets with the thumbs-up rate were selected as the analysis samples.

According to the official accounts, the top 50 tweets in terms of the thumbs-up rate include "What CUFE", "CUFE Student Affairs Office", "CUFE" and "CUFE Graduate Student Union.” However, "CUFE Bengdou” tweets, which rank high on the most-read list, do not appear in the thumbs-up top 50. To some extent, 
Content type structure of the top 50 most-read Tweets

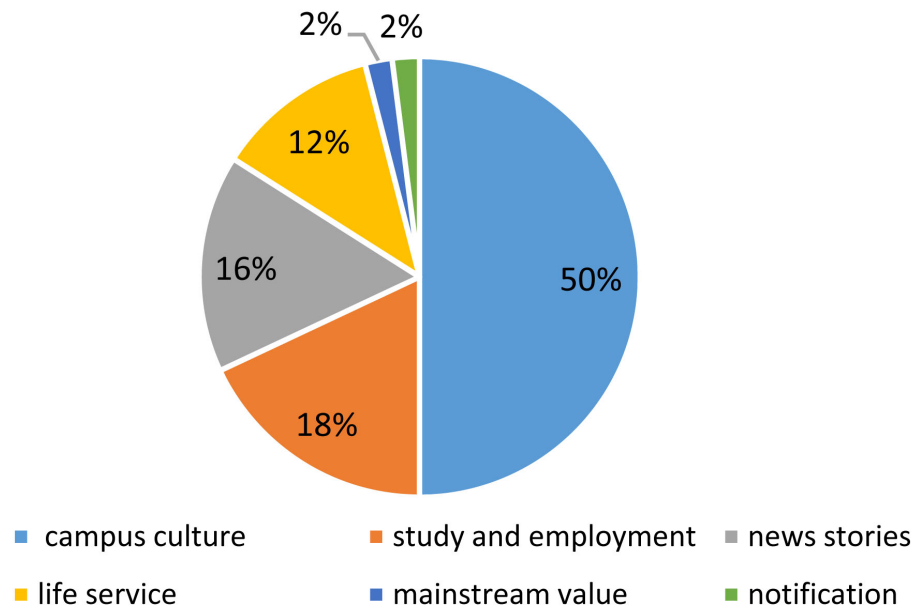

Figure 2. Content type structure of the top 50 most-read tweets.

this finding shows that students at CUFE have a lower recognition for articles published by "CUFE Bengdou" than other public accounts. After analyzing the sections of the public account "CUFE Bengdou", such as postgraduate entrance examinations, real exam questions, trampoline Taobao, and rental graduation clothes, the research team found that the possible reason was that the tweets of "Zhicai Trampoline" as a public account used for commercial purposes generally had a stronger inclination toward seeking commercial profit. Most of the tweets themselves are not original and do not express explicit opinions. Readers do not have an emotional reaction or value judgments of the tweets while reading them; thus, they do not experience emotional communication or value resonance. Therefore, it is difficult for such tweets to stimulate readers to perform "thumbs-up" actions (Wang, Zhao, \& Wang, 2015).

According to the proportion of the public accounts of the top 50 tweets in terms of the thumbs-up rate, there are 31 "What CUFE" tweets, accounting for $62 \%$, followed by the "CUFE Student Affairs Office" category with eight tweets (16\%), seven "CUFE Graduate Student Union" tweets (14\%), and four tweets from "CUFE" (8\%), as shown in Figure 3.

The tweet content types consist of five categories, including news reports, campus culture, study and employment, life services, and mainstream ideology, but not notification or voting. Among them, there are 15 news reports (30\%), 12 concerning mainstream values (24\%), 11 on campus culture (22\%), eight on life services (16\%), and four studies on employment (8\%).

By analyzing the content types, we can see that students are more likely to make clear value judgments on news articles. In particular, we should pay attention to the large proportion of tweets about mainstream values, which indicates that college students are not as opposed to articles about mainstream values and education as previously thought. Through further analysis of the mainstream 


\section{The proportion chart of the official accounts of the top 50 twitter sources with thumb up rate}

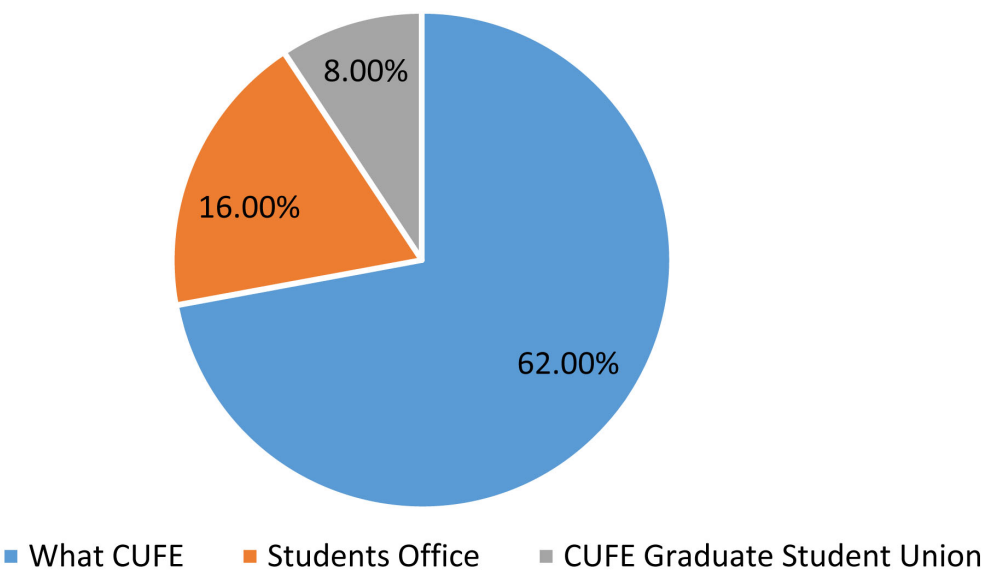

Figure 3. The proportion chart of the official accounts of the top 50 twitter sources with the thumbs-up rate.

value education articles, which account for up to $24 \%$, we can find that these articles mainly involve the spirit of volunteerism, a focus on the two sessions, advanced figures, and so on. In other words, the ideological consciousness of the students at CUFE is relatively positive, which is recognized and in line with mainstream values (Lovejoy \& Saxton, 2012).

Regarding the form of tweets, the tweets related to articles with a high rate of likes mainly adopt the text + picture form. Compared with the various forms of highly read articles (e.g., audio and video), the presentation form of highly liked tweets is somewhat monotonous. Thus, it can be seen that diversified forms of tweets can attract students to click and read, as well as improve the reading quantity of articles, but this approach does not necessarily result in positive feedback from students (Vickie, 2015). Furthermore, the influential factor that makes students identify with an article is the content of the article.

\subsection{The Relationship between Publication Time and Reading Amount}

As shown in Table 3, all tweets were counted according to the time of publication. By analyzing the relationship diagram between the release time of tweets and the number of tweets read, it can be seen that, from the time node, tweets published at 9:00 am, 12:00 noon, and 14:00 are read the largest number of times. In terms of time distribution, the number of tweets posted during the day is higher than that at night. However, from the perspective of college students' full-day activities, this is not a positive phenomenon because students have classes and must study during the daytime. This finding may reflect the widespread phenomenon of students using WeChat in class and study periods. 
Table 3. Statistical table of publication volume at different time periods throughout the day.

\begin{tabular}{ccc}
\hline Tweet times & Number of articles & Average number of readings \\
\hline $07: 00$ & 20 & 337 \\
$08: 00$ & 185 & 588 \\
$09: 00$ & 55 & 2508 \\
$10: 00$ & 54 & 1431 \\
$11: 00$ & 41 & 1966 \\
$12: 00$ & 30 & 3530 \\
$13: 00$ & 38 & 1630 \\
$14: 00$ & 49 & 2407 \\
$15: 00$ & 59 & 1745 \\
$16: 00$ & 63 & 2346 \\
$17: 00$ & 96 & 1549 \\
$18: 00$ & 123 & 2031 \\
$19: 00$ & 147 & 1123 \\
$20: 00$ & 793 & 725 \\
$21: 00$ & 549 & 1290 \\
$22: 00$ & 646 & 1218 \\
$23: 00$ & 225 & 1381 \\
$00: 00$ & 14 & 1930 \\
$01: 00$ & 2 & 1251 \\
\hline & & \\
\hline & & \\
\hline
\end{tabular}

\section{Conclusion}

To sum up, college students frequently use public WeChat accounts. The five popular public accounts at CUFE maintained a high degree of activity throughout 2019, with a high degree of reading and interaction with tweets. This finding indicates that college students pay the highest amount of attention to news reports and employment information on campus life and study, and WeChat has become the main channel for college students to obtain campus information. Tweets with rich forms and high content relevance are obviously more likely to attract the attention of college students. These conclusions lay an empirical foundation for the network education of college students.

\section{Funding}

This paper is a phased achievement of the key project of the Beijing Social Science Foundation, "Research on collaborative development of e-commerce in Beijing, Tianjin, and Hebei” (No. 15JDJGD093); a special project of ideological and political education for college students in Beijing "Research on content optimization of network ideological and political education in universities based on data mining" (No. BJSZ20182X41); Beijing Outstanding Young Scientist Program (BJJWZYJH01201910034034); supported by the 111 Project (B20094); the Program for First-class Discipline Construction in Central University of Finance and Economics; the Beijing Program for "Double First-Class" Construction Project of Central Universities. 


\section{Conflicts of Interest}

The authors declare no conflicts of interest regarding the publication of this paper.

\section{References}

Lin, X., \& Mou, J. (2019). Social Media Fatigue-Technological Antecedents and the Moderating Roles of Personality Traits: The Case of WeChat. Computers in Human Behavior, 101, 297-310. https://doi.org/10.1016/j.chb.2019.08.001

Lovejoy, K., \& Saxton, G. D. (2012). Information, Community, and Action: How Nonprofit Organizations Use Social Media. Journal of Computer-Mediated Communication, 17, 337-353. https://doi.org/10.1111/j.1083-6101.2012.01576.x

Matthews, W. J. (2013). Relatively Random: Context Effects on Perceived Randomness and Predicted Outcomes. Journal of Experimental Psychology. Learning, Memory, and Cognition, 39, 1642-1648.

Small, H. (2014). Co-Citation in the Scientific Literature: A New Measure of the Relationship between Two Documents. Journal of the American Society for Information Science \& Technology, 24, 265-269. https://doi.org/10.1002/asi.4630240406

Teresi, J. A. (2016). Data Sharing in the New Real-World. Medical Care, 12.

Tyrer, C. (2019). Beyond Social Chit Chat? Analyzing the Social Practice of a Mobile Messaging Service on a Higher Education Teacher Development Course. Springer International Publishing, 16, Article Number: 13.

https://doi.org/10.1186/s41239-019-0143-4

Vickie, C. (2015) Motivation to Participate in an Online Citizen Science Game. Science Communication, 37, 723-746. https://doi.org/10.1177/1075547015609322

Wang, H. Y., Wang, J., Ding, L. T., \& Wei, W. (2017). Knowledge Transmission Model with Consideration of Self-Learning Mechanism in Complex Networks. Applied Mathematics and Computation, 304, 83-92.

Wang, Z., Zhao, J., \& Wang, Y. (2015), Social Networks in Marketing Research 2001-2014: A Co-Word Analysis. Scientometrics, 105, 65-82. https://doi.org/10.1007/s11192-015-1672-9

Zhang, W. L., \& Zhang, P. (2017). Discussion on University Students' Information Acceptance, Identification Differences and Causes of WeChat Public Account. Modern Communication, 12, 78 .

Zhao, R. Y., \& Wei, M. K. (2017) Academic Impact Evaluation of Wechat in View of Social Media Perspective. Scientometrics, 112, 1777-1791. https://doi.org/10.1007/s11192-017-2439-2

Zhu, H.-M., Zhang, S.-T., \& Jin, Z. (2016). The Effects of Online Social Networks on Tacit Knowledge Transmission. Physica A: Statistical Mechanics and Its Applicat., 441, 192-198. 